\title{
Perspectivas históricas de la Investigación Operacional
}

\author{
Operational Research Historical Perspectives
}

\author{
Cristiane Hengler Corrêa Bernardo* \\ ORCID iD 0000-0002-9957-7437 \\ Viviane Hengler Corrêa Chaves** \\ ORCID iD 0000-0003-2848-4505 \\ Ricardo César Gonçalves Sant'Ana*** \\ ORCID iD 0000-0003-1387-4519 \\ Marta Pagán Martínez**** \\ ORCID iD 0000-0001-7363-2939
}

\begin{abstract}
Resumen
La historia de la Investigación Operacional se inserta en la historia de los grandes acontecimientos tecnológicos y científicos del siglo XX, y hacer una reconstrucción histórica de su surgimiento y evolución, objetivo de este artículo, es una manera de entender, además del desarrollo de la matemática aplicada, la movilización de la ciencia durante la Segunda Guerra Mundial y los contextos social y político que la originaron. Otra vertiente de esa historia es su influencia en el surgimiento de otras ciencias y aplicaciones, cuyos desdoblamientos durante la posguerra contribuyeron al aumento de la racionalización de la sociedad moderna. El objetivo de la investigación no es detallar sus técnicas, sino entender sus orígenes y desarrollo, cuyo éxito es atribuido a la práctica interdisciplinar. Los resultados de la investigación posibilitan la discusión de temas tales como la interdisciplinariedad; interacciones entre resolución de problemas prácticos e investigación; valorización de la matematización y globalización de procedimientos y métodos; modelado matemático y, por fin, cómo el conocimiento científico puede ser moldeado por los intereses políticos y sociales.
\end{abstract}

Palabras clave: Historia. Investigación Operacional. Segunda Guerra Mundial.

\footnotetext{
* Doutora em Educação pela Universidade Federal do Mato Grosso do Sul (UFMS). Professora Assistente Doutora da Faculdade de Ciências e Engenharia da Universidade Estadual Paulista "Júlio de Mesquita Filho" (UNESP) e do Programa de Pós-Graduação em Agronegócio e Desenvolvimento da UNESP, Tupã, São Paulo, Brasil. Endereço para correspondência: Avenida Domingos da Costa Lopes, 780, Jd. Itaipu, Tupã, São Paulo, Brasil, CEP: 17602-496. E-mail: cristiane@ tupa.unesp.br.

** Doutora em Educação Matemática pela Universidade Estadual Paulista "Júlio de Mesquita Filho" (UNESP). Professora Substituta do Instituto de Geociências e Ciências Exatas da Universidade Estadual Paulista "Júlio de Mesquita Filho" (UNESP), Rio Claro, São Paulo, Brasil. Endereço para correspondência: Avenida 24A, 1515, Bela Vista, Rio Claro, São Paulo, Brasil, CEP: 13506-900. E-mail: viviane.hcc@ bol.com.br.

*** Doutor em Ciências da Informação pela Universidade Estadual Paulista "Júlio de Mesquita Filho" (UNESP). Professor Assistente Doutor da Faculdade de Ciências e Engenharia da Universidade Estadual Paulista "Júlio de Mesquita Filho" (UNESP), Tupã, São Paulo, Brasil. Endereço para correspondência: Avenida Domingos da Costa Lopes, 780, Jd. Itaipu, Tupã, São Paulo, Brasil, CEP: 17602-496. E-mail: ricardosantana@ tupa.unesp.br.

**** Doutora em Técnicas e Métodos Atuais em Informação pela Universidade de Murcia na Espanha. Atualmente realiza pós-doutorado na Universidade Federal do Mato Grosso do Sul (UFMS), Campo Grande, Mato Grosso do Sul, Brasil. Endereço para correspondência: Avenida Senador Filinto Muller, 932/933, Vila Ipiranga, Campo Grande, Mato Grosso do Sul, Brasil, CEP: 79074-460. E-mail: pagán.marta@ gmail.com.
} 


\begin{abstract}
The history of Operational Research is part of the history of great technological and scientific developments of the twentieth century and make a historical reconstruction of its emergence and evolution, objective of this paper, as one way to further understand the development of applied mathematics, the mobilization of science during the Second World War, and the social and political contexts from which it was originated. Another aspect of this history is its influence on the development of other sciences and applications, which split the post-war period and contributed to the increased rationalization of modern society. The purpose of this research is not detailing its technique, but understanding its origins and development, which success is attributed to the interdisciplinary practice. The results of the research made it possible to discuss topics such as interdisciplinarity; interactions between practical problem solving and research; appreciation of mathematization and globalization of procedures and methods; mathematical modeling and, finally, how scientific knowledge can be shaped by political and social interests.
\end{abstract}

Keywords: History. Operational Research. Second World War.

\title{
1 Introducción
}

La Segunda Guerra Mundial fue un divisor de aguas para la práctica de la ciencia, y un marco de cambios en varios sectores del conocimiento. Se puede destacar, entre estos cambios, el desarrollo tecnológico que permeó todo el siglo XX y que, al finalizar la guerra, impulsó la evolución de los computadores para fines comerciales. Las innovaciones conquistadas en este período propiciaron la automatización de la recolección, almacenamiento y recuperación de datos, principalmente a través de los recursos digitales, transformando dispositivos en verdaderas extensiones de competencias humanas, tal como las palancas amplían la fuerza aplicada, las tecnologías informacionales pasaron, entonces, a potenciar las capacidades informacionales humanas (SERRES, 2003).

La investigación operacional (IO) es fruto de la práctica de esta ciencia, cuya historia indica cómo el dominio de las matemáticas evolucionó mediante las interacciones entre resolución de problemas prácticos e investigación. La IO surgió un poco antes de la Segunda Guerra Mundial como forma organizada de investigación y, como su propio nombre evidencia, la investigación operacional es investigación y produce investigación (HILLIER; LIEBERMAN, 2013).

Su historia indica los caminos trazados por sus avances y conquistas, y, de ese modo, permear esa trayectoria puede permitir reflexiones que apunten a las aplicaciones para las cuales fueron utilizadas y para las que el futuro aún revela ante las actuales demandas de la sociedad globalizada. En este sentido, este artículo tiene como objetivo trazar un paralelo entre la evolución de la IO, el desarrollo de las matemáticas aplicadas, la movilización de la ciencia durante la Segunda Guerra Mundial y los contextos social y político que la originaron. 
Para ello, este artículo aborda el período histórico que va desde el surgimiento de la IO, desde mediados de 1935 hasta 1950, período en que, de acuerdo con Arenales et al. (2015), estaban consolidados los conceptos de la IO y de sus principales métodos: la Programación Lineal y la Teoría de los Juegos.

Las investigaciones históricas realizadas sobre la Investigación Operacional (IO) por Ackoff e Sasieni (1971); Henderson (1966); Mirowski (1999); Miguel (2006), de entre otras presentadas a lo largo del texto y propuestas para ser descritas en el presente artículo, apuntan sus contribuciones a los esfuerzos de guerra, sus influencias en el surgimiento de otras ciencias y aplicaciones, y las tendencias durante la posguerra de la II Guerra Mundial (1945-1947).

De acuerdo con Mirowski (1999), detrás de esta historia también se puede notar el surgimiento de la racionalidad social, abastecida por la ciencia y la tecnología. También se constata que el conocimiento científico puede ser moldeado por los intereses políticos y sociales.

\section{La investigación operacional y su contexto histórico}

La investigación operacional tuvo sus orígenes fundados en las fuerzas armadas de los Estados Unidos de América y Gran Bretaña durante la Segunda Guerra Mundial, cuando entonces la estadística se utilizaba para determinar cuáles tácticas militares funcionaban. La lógica pragmática de las operaciones militares especificaba las agendas de investigación y, en ese contexto, lo que importaba era la eficacia del método, pues las soluciones tenían que ser alcanzadas en tiempo hábil (HILLIER; LIEBERMAN, 2013; MIROWSHI, 1999).

De este modo, la investigación operacional se inserta en la historia de los grandes acontecimientos tecnológicos y científicos del siglo XX. Las aplicaciones matemáticas desempeñaron un papel fundamental durante ese período, puesto que instrumentalizaron la física moderna y otras ciencias, y el éxito de sus aplicaciones, con fines puramente militares, puso en evidencia a los matemáticos, que distribuían las credenciales de los nuevos espacios que surgieron para la práctica de la ciencia como consecuencia de la confluencia entre los militares y los científicos (MIROWSHI, 1999; MIGUEL, 2006).

Otro factor relevante fue la utilización de modelos lógicos para la práctica de la ciencia, cuya notoriedad fue alcanzada por su uso en la física cuántica, que tenía elevado prestigio en la época, debido a la imposibilidad de analizar los resultados mediante datos experimentales (HILLIER; LIEBERMAN, 2013; MIROWSHI, 1999). 


\subsection{Primeros ensayos}

Bajo el punto de vista histórico, es posible identificar los orígenes de la Investigación Operacional en épocas más remotas, pero es difícil encontrar, precisamente, el hecho histórico que la originó (MCARTHUR, 1990; TREFETHEN, 1966; Morse; Kimball (2012); GLICK; CHARTERS, 1983; FORTUN; SCHWEBER, 1993).

Mirando hacia el pasado, no en busca de un origen de la IO, pero sí del seguimiento de sus manifestaciones, se encuentra en el trabajo del físico y matemático griego Arquímedes, uno de sus precursores. Arquímedes idealizó la defensa de la ciudad de Siracusa, proyectando nuevas armas y tácticas militares basadas en sus estudios científicos (TREFETHEN, 1966). Muchos siglos después comenzaron a surgir los primeros problemas que serán abrazados por la IO.

La Revolución Industrial reveló una nueva era que, aliada a las innovaciones tecnológicas, impulsó el crecimiento en las industrias y generó un aumento sin precedentes en la productividad del trabajo. Para atender a las necesidades y exigencias de esa nueva sociedad, surgieron nuevos tipos de administración, nuevas industrias, nuevas máquinas, y estudios destinados a aumentar la eficiencia de determinadas operaciones industriales (HILLIER; LIEBERMAN, 2013).

Pese a los primeros ensayos que pueden ser relacionados con la IO y que son presentados en las siguientes secciones, el término Investigación Operacional es relativamente nuevo y de origen militar, siendo usado, por primera vez, en Gran Bretaña, un poco antes de la Segunda Guerra Mundial, en 1938, e relacionado a investigaciones asociadas al radar (KIRBY, 2003).

En ese contexto, lo importante es que se conozca el escenario en que fue creada y desarrollada. Se percibe que ese contexto es bastante complejo y el surgimiento y desarrollo de la investigación operacional es relatado por autores en diversas perspectivas y fases distintas, de acuerdo con los países en que se presenta. Se destaca, además, que no hay consenso sobre la fecha exacta del surgimiento de la investigación operacional, ya que su desarrollo ocurre paralelamente en varios países y con aplicaciones distintas. Para ese artículo, se buscó relacionar ese surgimiento a las estrategias de defensa en fase de las grandes guerras.

\subsection{Inglaterra: el inicio de la Investigación Operacional}

En 1914, tiene inicio la Primera Guerra Mundial. En Europa y América del Norte surgen tentativas para analizar matemáticamente las operaciones militares. En Inglaterra, Lanchester, 
como pasatiempo, hacía ensayos sobre victoria, poderío de fuego, y otras simulaciones por medio de modelos matemáticos. En Inglaterra, en 1934, fue creado el Comité para el Estudio Científico de la Defensa Aérea, cuya propuesta era la utilización de los recursos tecnológicos para reforzar y modernizar los métodos utilizados por la Defensa Aérea contra los aviones enemigos. Un año después de la creación de ese comité, surge el radar. Previendo su utilización en las tácticas de defensa empleadas y en la dirección y control de su flota, el Comité realizó experimentos simulados de detección de aviones enemigos y el envío de los suyos para interceptarlos, lo que posibilitó el análisis y el desarrollo de nuevos métodos de interceptación (TREST, 1998; TREFETHEN, 1966).

Para Trefethen (1966), esos experimentos originaron la formación de equipos, constituidos por científicos y militares, para trabajar con actividades de esa naturaleza. Un poco antes del inicio de la Segunda Guerra Mundial, en el puesto de investigación de Bawdsey, en Inglaterra, ya existía un equipo trabajando con esas actividades, cuyo objetivo era localizar objetos en el espacio por medio de la nueva tecnología de radar. Albert Percival Rowe era el superintendente de ese puesto y, debido a su iniciativa, se produjo esa aproximación entre los científicos británicos y los militares, con el objetivo de enseñarlos a usar el radar.

G.A. Roberts era el científico encargado de estudiarlo e integrarlo al sistema ya existente (ROWE, 2015; TREFETHEN, 1966). El comandante R. Hart y A.P. Rowe estaban organizándose para que, cuando se iniciara la guerra, fuera transferido un grupo de científicos de ese puesto para el Cuartel General del Mando de Combate de la Real Fuerza Aérea, con el objetivo de crear, en Stanmore, un puesto de IO (TREFETHEN, 1966; ROWE, 2015).

Simultáneamente, E.C. Williams desarrollaba estudios importantes para la Royal Air Force (RAF), de los cuales se destaca el análisis de las variaciones verificadas en los resultados presentados por las estaciones de alarma aérea. Percibiendo la semejanza entre los trabajos de Roberts y Williams, Rowe los reunió en el sector de Establecimiento de Investigaciones de Telecomunicaciones, que era supervisado por H. Larnder. Ese fue el sector transferido para Stanmore, cuando la guerra comenzó (TREFETHEN, 1966; ROWE, 2015).

Como el propio Larnder pondera, ese sector pasa a analizar todos los fallos de interceptación de los aviones alemanes que buscaban adentrarse en la isla británica. A partir de esos informes de combate, surgen nuevas técnicas para el control de las formaciones británicas contra las germánicas, y también reglas simples, pero de resultados bastante eficientes, como, por ejemplo, la fragmentación de señales en el radar, que sería un indicador de que los bombarderos enemigos estaban perdiendo altitud para lanzar sus bombas (TREFETHEN, 1966; ROWE, 2015). 
En agosto de 1940, el general F.A. Pile, Comandante Supremo del Mando Antiaéreo, solicitó ayuda a los científicos para la utilización del equipamiento de radar en las posiciones de artillería. Tal equipamiento suministraba el ángulo de inclinación y la posición de un bombardero enemigo, por medio de una tecnología recientemente desarrollada, que propiciaba la lectura de su elevación. Sin embargo, ese equipamiento no garantizaba la misma performance en las posiciones de artillería que las observadas en las estaciones experimentales (RANGER, 1993).

Destacó, en el estudio de ese problema, el científico P.M.S. Blackett, que reunió para ese trabajo a un equipo multidisciplinar compuesto por tres fisiologístas, dos físicomatemáticos, un astrofísico, un oficial del ejército, un agrimensor, un físico y dos matemáticos. Para solucionarlo, fue necesario que las observaciones científicas fueran realizadas en las propias posiciones de artillería durante operaciones reales. Ese grupo de investigación de Blackett, creado en 1940, fue conocido como Blackett's Circus que ganó notoriedad cuando se aventuró por otra área que no era de su competencia: la táctica militar (MIROWSKI, 1999).

Blackett y algunos de los miembros de su equipo fueron transferidos, en 1941, a otro mando, en el cual se vieron envueltos en problemas relativos a la detección de navíos y submarinos, por medio de la utilización de radar. El éxito de esas operaciones provocó un aumento en la búsqueda de servicios de esa naturaleza y, tras el inicio de la guerra, los tres servicios militares británicos tenían equipos organizados de investigación operacional trabajando junto con los científicos. Considerado el padre de la IO, Blackett fue el primero en introducirla en el Ejército y en la Marina, haciéndose, en 1941, Consejero Científico y Director de la Investigación Operacional Naval. Fue él quien, indiscutiblemente, definió las reglas básicas por las cuales la IO debería operar y el primero en definir sus características (KIRBY, 2003; TREFETHEN, 1966).

La colaboración entre científicos y militares pasó a ser una constante durante ese periodo de guerra, cuya práctica acabó difundiéndose entre los aliados: Estados Unidos, Canadá y Francia. Esa unión se debe, según Ackoff y Sasieni (1971), al gran desarrollo de la tecnología militar ocurrido entre el fin de la Primera Guerra Mundial y el inicio de la Segunda. Los administradores militares al no conseguir aplicar esas nuevas tecnologías a las tácticas y a las estrategias de guerra, se ven confrontados con problemas de gran dimensión y complejidad. Ese desajuste los llevó a solicitar ayuda de los científicos, siendo una de ellas la incorporación de la nueva tecnología de radar a sus tácticas y estrategias de guerra (MIROWSKI, 1999).

La proximidad dio como resultado la creación de un nuevo espacio para la práctica de la ciencia, tornando la IO tan importante después de la Segunda Guerra, debido a la ampliación 
de la relación entre científicos e Estado. Se abrió un espacio para muchas reformulaciones, incluso en la organización y estilo de investigación, debido a la diversidad de métodos que surgieron en aquel periodo (HENDERSON, 1966).

Bawdsey es considerado como el lugar de nacimiento de la Investigación Operacional, en el periodo comprendido entre 1935-1938, y los ingleses, los gestores de su concepto básico. Reflexionando sobre las actividades de investigación que la originaron y en su conducción práctica, hoy parecen ejemplos inadecuados de IO (TREFETHEN, 1966).

Es siempre la historia la que cuenta lo que pasó, incluso los momentos trágicos de la guerra deben ser registrados, pues mañana existirá la oportunidad de replantearlos y, tal vez, permitir a la humanidad entera una segunda oportunidad. Sin embargo, hoy se pueden presumir las dificultades para documentar esas operaciones. En la realidad, en muchos casos, "apenas había tiempo para el registro de las actividades diarias que simplificara la recolección de la materia y que, posteriormente, serviría para la preparación de la Historia de la Investigación Operacional" (TREFETHEN, 1966, p. 27).

A pesar de esas dificultades, fue posible delinear claramente algunas de sus características. Se hizo obvio, por ejemplo, que la organización constituida en una oficina central y las unidades de campo se adecuasen mejor a la situación. En la oficina central eran efectuados el procesamiento y la planificación de los datos, desde donde eran realizados los contactos con los ministerios. En las unidades de campo eran realizadas las observaciones y evaluaciones, esenciales para el estudio de los problemas. Otra característica relevante para su éxito, fue la diversidad en la formación de los equipos. El uso de equipos heterogéneos disponibiliza un mayor arsenal de técnicas de investigación y ayuda a construir la conexión entre la teoría y la práctica. También son características que la distinguen de las primeras actividades de investigación, durante la Segunda Guerra Mundial, su alcance sistémico (JOHNSON, 1966).

El surgimiento de la cibernética, durante ese periodo, refuerza esa concepción, en que la propia realidad es recortada en sistemas. Esa idea fue tomando forma y estructurando a toda la sociedad moderna en un mundo sistémico. Hoy, son construidos modelos computacionales reflejados en la naturaleza, con incontables sistemas extremadamente complejos y adaptables a los cambios del ambiente. Esas características satisfacen los requisitos necesarios para las nuevas generaciones de sistemas, en los cuales la idea de duplicar el éxito de la naturaleza, aunque sea en una pequeña parte, permite construir otros sistemas más sofisticados (CHAVES, 2011; MIROWSKI, 1999). 
La experiencia británica en la Segunda Guerra Mundial mostró que las personas que trabajaron con IO podían hacerlo mejor se estuvieran libres de las restricciones impuestas a los militares. Fue la libertad para comunicarse directamente con todos los implicados, de todas las clases, la que hizo posible el grado de eficiencia en investigación, esencial para la investigación científica. A pesar de la ciencia, en ese periodo, haber sido capturada por el complejo industrial militar, de cierta forma los científicos consiguieron preservar su autonomía intelectual, lo que para los iluministas es un ideal irrenunciable (FORTUN; SCHWEBER, 1993).

\subsection{EEUU y la Investigación Operacional}

Un año antes de que los Estados Unidos (EEUU) entraran en la Segunda Guerra Mundial, existía una profunda inseguridad por parte de los científicos americanos con relación al sistema de defensa vigente en el país, debido al débil poder de la tecnología bélica, a la falta de planificación para priorizar las estrategias y a la necesidad de una alineación tecnológica militar (MCARTHUR, 1990).

Mcarthur (1990) afirma que presintiendo un conflicto inevitable y su dimensión real, cuatro líderes de la ciencia americana se reunieron para discutir el asunto, pretendiendo adecuarse a las necesidades de la guerra moderna. Esos científicos eran Vannevar Bush, presidente del Carnegie Institution of Washington, un ingeniero eléctrico, Karl Taylor Compton, presidente del Massachusetts Institute of Technology (MIT), un físico, James Bryant Conant, presidente de Harvard University, un químico y Frank Baldwin Jewett, ingeniero eléctrico y presidente de la Academia Nacional de Ciencias y de la Bell Telephone Laboratories.

Vannevar Bush estimuló ese debate resultante del que había vivenciado durante la Primera Guerra Mundial, cuando la falta de cooperación entre científicos civiles y militares fue crítica. Bush fue un intelectual y creador de políticas de incentivo y su liderazgo en el desarrollo del complejo militar americano tenía como plan de acción la incorporación del conocimiento científico, por medio de la participación de los científicos en los esfuerzos de guerra (MCARTHUR, 1990).

Como portavoz de ese grupo, Bush convenció al presidente Roosevelt de la necesidad de movilizar la investigación científica en el campo militar, y mostró que la tecnología era la llave para la victoria. Así, con el apoyo del presidente Roosevelt, fue creado, el 27 de junio de 1940, el National Defense Research Committee (NDRC), con presupuestos para el rearme y la financiación de la ciencia. Esa organización coordinó, supervisó y realizó investigaciones 
científicas, iniciando los estudios de las tecnologías más importantes de la Segunda Guerra Mundial - la bomba atómica y el radar (DUREN et al., 1989).

Un año después, el 28 de junio de 1941, fue creado el Office of Scientific Research and Development (OSRD), agencia del gobierno federal, que acabó incorporando el NDRC y coordinando las actividades científicas surgidas durante la guerra. Con recursos y fondos ilimitados, la investigación y el desarrollo eran efectuados por las Universidades y por las Instituciones Industriales, por medio de contratos firmados con el OSRD. La oficina fue organizada como es, hoy, la National Science Foundation (NSF), creada inmediatamente después del término de la guerra, también por recomendación de Vannevar Bush al presidente (DUREN et al., 1989; MCARTHUR, 1990).

Al final de la guerra, el NDRC estaba constituido por diecinueve divisiones, dos paneles de matemática aplicada y tres comisiones. En esa organización, las divisiones consideradas eran: investigación balística, guerra submarina, radar, proyectiles especiales, y otras. El Panel de Matemática Aplicada (AMP) se dividía en la sede Céntrica y en el Grupo de Investigación Estadística (SRG) (TREFETHEN, 1966; DUREN et al., 1989).

Semejante a la Fuerza Aérea Británica, la de Estados Unidos también había iniciado sus estudios sobre los problemas relativos a la nueva tecnología de radar, teniendo científicos al frente de sus mandos operacionales. En el transcurrir de la guerra, aproximadamente cuatrocientas personas trabajaron con IO para las Fuerzas Armadas, como analistas operacionales. Entre ellos había oficiales, soldados, analistas y civiles (TREFETHEN, 1966).

Verificándose que no existía ninguna investigación para orientar el perfeccionamiento de las bombas, destinados a las operaciones ofensivas, cerca de cincuenta miembros del Laboratorio del Arsenal de la Marina, liderados por el científico Ellis A. Johnson, iniciaron un estudio sistemático y minucioso de todos los problemas de orden táctica, estratégica y técnica, referentes a ese tema. Usaron la técnica del juego de guerra para perfeccionar modelos de operaciones posibles y probar diversas tácticas y armas de guerra. Ese trabajo justificó la creación de un grupo de IO del Laboratorio del Arsenal de la Marina, en marzo de 1942 (TREFETHEN, 1966).

La investigación operacional militar pasó, además de resolver sus problemas tácticos, a encontrar soluciones óptimas para los problemas emergentes de la situación y, también, a formular previsiones. Ese nuevo abordaje para solucionar problemas, subordinados a la racionalización de las actividades militares, dio inicio a un proceso de reconfiguración de las estructuras de defensa de los Estados Unidos por las vías de una concepción científica de los estudios estratégicos (MIROWSKI, 1999). 
En consonancia con Mirowski (1999, p. 688) la definición de la IO dada por Ellis A. Johnson y Patrick Blackett, tras su surgimiento, es la de ser una "previsión y comparación de valores, efectividad y costes de un conjunto de cursos alternativos propuestos de acción envolviendo el sistema hombre-máquina".

Debido a las intensificaciones de la guerra submarina, en abril de 1942, el Comandante de la Unidad de Guerra Antisubmarina solicitó al Coordinador de Investigación y Desarrollo la formación de un grupo de IO. En mayo de 1942, fue organizada una sección con siete investigadores de la Universidad de Columbia y para dirigir ese equipo fue convocado Philip M. Morse, físico del MIT (BARROS, 2004).

Ese grupo, inicialmente, trabajó en el análisis de los resultados de ataques navales y aéreos contra los submarinos enemigos, con el objetivo de mejorar la eficiencia de esas operaciones. En julio de 1943, ese grupo, ahora seis veces mayor, fue incorporado al Estado Mayor de la Décima Flota, como Grupo de Investigación Operacional de Guerra Antisubmarina y, al final de la guerra, abarcaba setenta y tres científicos con las más diversas experiencias, alternándose en los trabajos de campo y actuando en varios mandos (TREFETHEN, 1966; MIROWSKI, 1999).

Los problemas abordados por la IO, durante ese periodo, fueron tantos y tan variados que sería imposible relacionarlos. Otros importantes y aún no mencionados fueron: la relación de peso de las bombas, la precisión de bombardeos, las maniobras de navíos para evitar kamikazes y dimensión de los convoyes. En Estados Unidos, la IO recibió varias denominaciones: análisis de operaciones, evaluación de sistemas, investigación de operaciones, análisis de sistemas, ciencia de administración e investigación operacional (TREFETHEN, 1966).

Al volver a ver su historia, son encontradas algunas especificidades de su génesis. Primero, eran los científicos naturales, principalmente los físicos, que fueron los responsables por su creación, conducción y codificación, durante el régimen de la Segunda Guerra Mundial. Segundo, los militares buscaron ayuda de los civiles, en particular, de los científicos, para dirigir la guerra. Sus aplicaciones se restringieron a las militares y las agendas de investigaciones eran determinadas por sus necesidades. Tercero, se creía que existía un método científico genérico, el cual era utilizado por los investigadores operacionales en la resolución de esos problemas. Tras la construcción de la bomba atómica dicho método fue cuestionado, y todo pasó a ser una cuestión de posesión de conocimientos matemáticos (TREFETHEN, 1966; MIROWSKI,1999).

Es así como la IO es presentada en los documentos contemporáneos y en algunos relatos históricos de ese tiempo y, en vez de considerarla como parte de la matemática, una 
caracterización mejor sería pensar que algunos matemáticos fueron reclutados durante la Segunda Guerra Mundial para trabajar en unidades multidisciplinares, comandados por los físicos y conducidos a participar de las más diversas actividades, agrupadas bajo la rúbrica de IO (MIROWSKI, 1999).

Tras el inicio de la guerra, muchos matemáticos fueron invitados a involucrarse más con las necesidades de los militares, a fin de resolver problemas relacionados con el esfuerzo de guerra. Fue creado, en 1942, el Applied Matematics Panel (AMP), como una división de la NDRC, para ayudar a resolver ese tipo de problema. Su objetivo era establecer grupos de matemática aplicada en varias Universidades, cuyos contratos serían firmados por la sede de la AMP, localizada en Manhattan, que suministraría los proyectos a ser analizados en respuesta a los pedidos de los militares o de sus contratadas. El AMP fue dirigido por el matemático Warren Weaver. En 1948, Weaver escribió un artículo con el título Ciencia y Complejidad, que abordaba temas como Ciencia y Guerra (TREFETHEN, 1966; DUREN et al., 1989; MIROWSKI, 1999).

En la primavera de 1942, Warren Weaver creó el Statistical Research Group (SRG) fundado en la Universidad de Columbia y soportado por la AMP. Ese grupo fue liderado por Harold Hotelling y formado por ingenieros, estadísticos y matemáticos. Sus tareas, inicialmente, tenían una estrecha semejanza con aquellas realizadas en las unidades británicas de IO, y muchos de sus problemas fueron resueltos por la calculadora electrónica, ya disponible en esa época. De los 572 informes y memorandos producidos por la SRG, la idea que atrajo más interés y desarrollo en el periodo de posguerra fue la teoría del análisis secuencial de Abraham Wald (DUREN et al., 1989).

Todavía, en la Universidad de Columbia, en marzo de 1943, fue creado el grupo Applied Mathematics Group at Columbia (AMG-C) y que se destacó por la importancia de los trabajos realizados (DUREN et al., 1989). En 1942, ya había como mínimo dos grupos activos de IO en EEUU, uno llamado Antisubmarine Warfare Operations Research Group (ASWORG) y el otro Radiation Laboratory del MIT. El Ilustre miembro de ambos grupos, desde el inicio, el matemático John von Neumann, fue uno de los científicos responsables de la forma en que la IO fue adoptada en América, en el sentido de hacerla una preocupación en los centros de investigación (MCCLOSKEY, 1987; MORSE, 1986).

John von Neumann (1953) y el economista, Oskar Morgenstern (1953) escribieron un libro, publicado por la Princepton University, en 1944, con el título "Teory of Games and Economic Behavior" (Teoría de los Juegos y Comportamiento Económico). La idea central es 
que siempre existe una solución racional para un conflicto bien definido entre dos individuos y cuyos intereses sean completamente opuestos.

Ese trabajo, de enfoque matemático, trata sobre la lógica de las situaciones de conflicto, además, de presentar soluciones y paradojas que ayudan a comprender mejor las limitaciones de la racionalidad humana. Neumann y Morgenstern presentaron su trabajo, la Teoría de los Juegos, como una ramificación de la IO, para la cual contribuyó la matemática, volcada en las preocupaciones estratégicas de la guerra.

Durante y después de la guerra, von Neumann fue consultor del Laboratorio de Investigación Balística, en el Aberdeen Proving Grounds, en Maryland. En esa unidad, realizaba estudios sobre balística, y su problema en ese proyecto era la gran cantidad de cálculos que tenían que ser efectuados. En el verano de 1944, Campbell (2004) relata que von Neumann, por casualidad, conoció a Herman Goldstine en la estación ferroviaria local y lo escuchó hablar sobre el proyecto ENIAC (Electronic Numerical Integrator and Computer) en el cual trabajaba. Ese proyecto había sido financiado por el laboratorio de Investigación Balística, ante la necesidad de encontrar una manera más rápida de calcular las trayectorias balísticas, pues había muchas variables en juego, como distancia, peso, velocidad, dirección del viento, además de otras, lo que hacía el cálculo más complejo y demorado (MCCLOSKEY, 1987; MORSE, 1986; MIROWSKI, 1999).

Fue para J. Presper y John Mauchly, idealizadores del proyecto ENIAC, cuando John von Neumann se presentó. Neumann contribuyó con grandes ideas para la construcción del design de ese primer computador digital electrónico, y escribió los principios básicos para su arquitectura, la cual quedó conocida como la Arquitectura de von Neumann, que se convertiría en un modelo para las futuras generaciones de mainframes. También estableció lazos duraderos con el computador como herramienta y ejemplo de racionalización organizacional, y aprobó la programación lineal para varias organizaciones militares, así como suministró el puente para la Teoría de la Información. "Para von Neumann, IO fue un vasto campo de analogías matemáticas inexploradas; y esas analogías suministraron el puente conceptual sobre la cual físicos de varias especialidades podrían entrar en la teoría social” (MIROWSKI, 1999, p. 692-693).

Durante el régimen de la guerra, quedó evidenciada la importancia de la tecnología para la industria bélica. El Proyecto Manhattan y los efectos devastadores de las bombas atómicas hicieron con que el gobierno americano pasara a valorar mucho más la investigación científica y tecnológica como estrategia política y militar, y también como un termómetro para el desarrollo económico (MIROWSKI, 1999). 
En la secuencia se presenta una síntesis de los principales acontecimientos que marcan la historia de la investigación operacional.

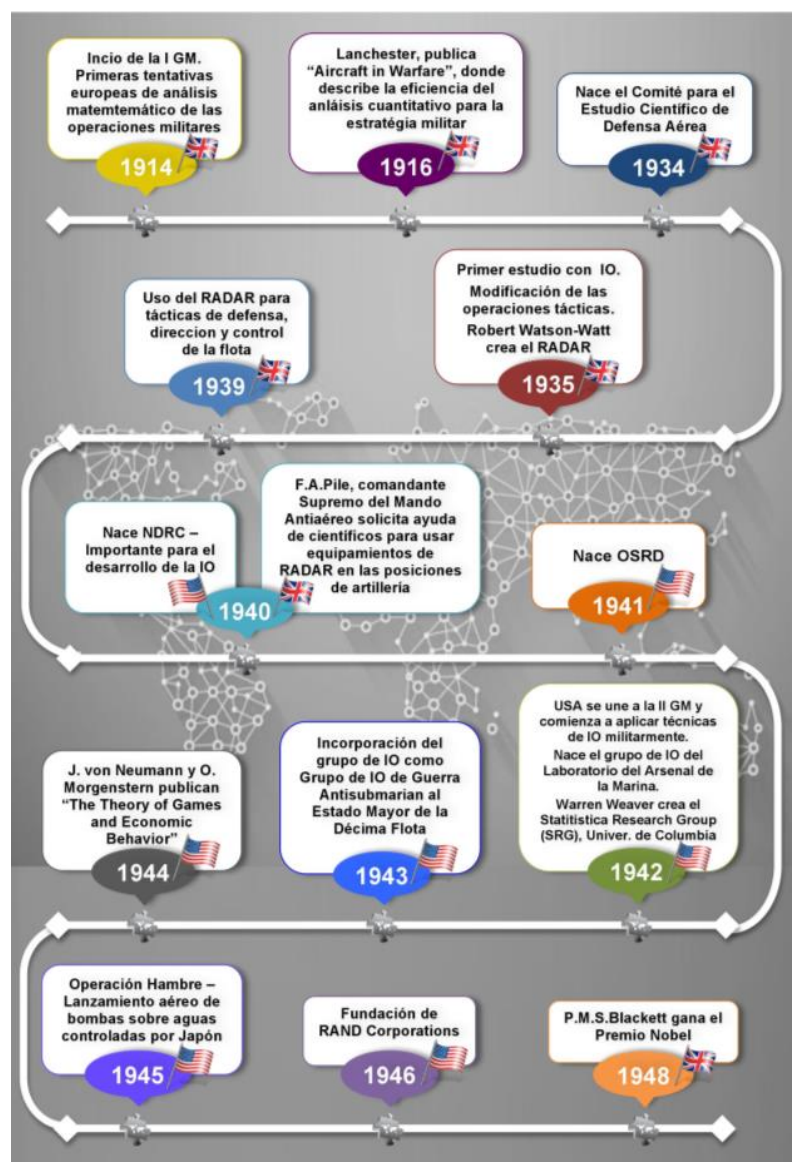

Figura 1 - Desarrollo de la Investigación Operacional en Inglaterra y EEUU Fuente: elaborado por los autores

\subsection{A Investigación Operacional tras el fin de la guerra}

Tras el fin de la guerra, la IO inglesa y la americana tomaron rumbos diferentes, y hay razones para creer que una de las causas haya sido el Movimiento para la Libertad de la Ciencia. Ese movimiento ocurrió en Inglaterra, en virtud de un grupo de intelectuales que se oponía a la interferencia militar en las actividades científicas. De entre esos intelectuales cabe destacar a Michael Polanyi, Friedrich von Hayek, John Baker y Arthur Tansley (TREFTHEN, 1966).

Los ingleses redujeron las inversiones en el área militar y, consecuentemente, liberaron a muchos especialistas que trabajaron con IO durante la guerra, los cuales fueron absorbidos por las industrias que necesitaban mano de obra para su reconstrucción. Inversamente a lo que aconteció en Inglaterra, en Estados Unidos aumentó la investigación en el sector militar y la IO se expandió aún más cuando la guerra terminó. Al final de la guerra, tanto el ejército americano como el inglés crearon divisiones permanentes de IO, en las cuales la mayor parte del trabajo 
realizado era clasificado como Secreto de Estado, no pudiendo ser revelada (CAMPBELL, 2004).

Antes de terminar las hostilidades de la guerra, algunos militares americanos querían dar continuidad al trabajo científico que estaba siendo realizado por los investigadores y científicos en el ámbito militar. Campbell (2004) afirma que un de esos militares fue el General Henry Harley Arnold, de la Fuerza Aérea, que, tras la guerra, tomó la iniciativa de crear un ambiente para preservar el espíritu innovador e intelectual que había sido establecido durante aquel régimen. Al reformular la planificación estratégica del Sector de Investigación y Desarrollo de la Fuerza Aérea, él resolvió subsidiar un proyecto que atendiera a sus expectativas y le proporcionara asistencia científica (BARROS, 2004; CAMPBELL, 2004).

De acuerdo con Campbell (2004), fueron invertidos diez millones de dólares de los fondos de la Fuerza Aérea Americana para financiar ese proyecto, denominado RAND, abreviatura de Research and Development, creado en diciembre de 1945. El General Arnold indujo a Donald Douglas, de Douglas Aircraft, para dirigirlo y, en marzo de 1946, la RAND pasa a ser una división de esa compañía. Según Campbell (2004), en 1948, ese proyecto se transformó en la RAND Corporation, una organización sin fines lucrativos y que fue, inicialmente, financiada por la Fundación Ford, transfiriéndose, así, desde Douglas Aircraft a su propia sede en Santa Mônica, California.

Quién idealizó la nueva sede para abrigar la RAND Corporation fue John Williams, del Grupo de Investigación Estadística de Princeton. La planeó de tal forma que su arquitectura aumentaría la probabilidad de que investigadores de varias áreas se encontraran durante sus actividades diarias. Ese cambio de experiencias entre personas de diferentes formaciones era una característica esencial para su funcionamiento, o sea, él quería hacer una réplica del ambiente de trabajo que tan bien había funcionado durante la guerra. Esa forma de organización, inmediatamente presentó datos concretos con el trabajo de Ed Paxson, que usó el término análisis de sistemas para describir el proceso que envuelve las operaciones militares (CAMPBELL, 2004).

El Análisis de Sistemas fue concebido en la RAND Corporation como un desarrollo de la IO. El cambio en el tiempo y en las definiciones de los problemas fue determinante para su surgimiento. En el periodo de posguerra había más tiempo para resolver los problemas y sus definiciones eran más detalladas y objetivas, al contrario de lo que ocurría durante el periodo de guerra. Otro aspecto a destacar es el trabajo del lógico matemático Albert Wohlstetter, que fue considerado uno de los más importantes analistas de la RAND y se convirtió en uno de los principales estrategas de seguridad nuclear del mundo (BARROS, 2004; CAMPBELL, 2004). 
Todo cambió drásticamente con la llegada de la Segunda Guerra Mundial. No sólo fueron renovadas las estructuras de financiación y organización de las ciencias físicas, sino también las concepciones económicas fueron irreversiblemente transformadas. Esa renovación de la definición de la ortodoxia económica americana se inició a partir del momento en que físicos y matemáticos innovaron con un conjunto de técnicas y herramientas para modelado de problemas de mando, control, comunicación y transmisión de información bajo la rúbrica de IO. Varios economistas neoclásicos fueron influenciados por estas técnicas y herramientas, las cuales fueron cruciales en la definición de la forma de la teoría económica de posguerra (MIROWSKI; SENT, 2002).

La RAND Corporation fue la incubadora de muchas otras producciones científicas, como: Inteligencia Artificial, Cibernética, Método Delphi, Programación Lineal, Programación Dinámica, Teoría de los Juegos etc. Y, en la década de 1950, ésta poseía la mayor instalación de computadores para fines científicos del mundo (MIROWSKI, 1999).

A partir de la Segunda Guerra Mundial, la ciencia entra en el umbral de una nueva era, cuando la mayoría de las soluciones para los problemas presentados pasa a contar con la ayuda de los computadores. Surgió una gran racionalidad dentro de las organizaciones, en gran medida debido a los métodos que comenzaron a ser empleados en el proceso decisorio de las empresas. La explosión industrial aumentó la complejidad de esas organizaciones, haciéndose más difícil ubicar los recursos disponibles de modo eficaz.

Ese nuevo linaje de problemas hizo que científicos y matemáticos desarrollaran nuevos modelos para atender a las necesidades administrativas, los cuales aluden a la decisión óptima y a los sistemas probabilísticos y determinísticos. La IO se centra en esa nueva clase de problemas, suministrando conclusiones precisas a los tomadores de decisiones y haciéndose uno de los principales soportes para la gestión en las organizaciones (MIROWSKI, 1999).

Las decisiones siempre estuvieron presentes en la evolución del hombre y siempre se reflejaron en la sociedad de la cual él forma parte, sin embargo, es a partir de 1945 cuando comenzaron a surgir varios estudios en esa área. La aplicación de la IO en la toma de decisiones se hizo viable en el transcurso del avance de la tecnología de los computadores, pues la gran mayoría de los modelos matemáticos desarrollados sería de poca utilidad si los cálculos tuvieran que ser realizados manualmente, haciendo imposible su aplicación (MIROWSKI, 1999).

Herbert A. Simon (1965) fue uno de los consultores de la RAND, y uno de los pensadores del proceso decisorio en las organizaciones. Su estudio fue un modelo en el campo de las Ciencias Sociales, cuyo foco yace en el estudio del comportamiento humano, en la toma de decisiones y en la resolución de problemas en las organizaciones. Actuó en varias áreas como 
psicología, inteligencia artificial, ciencia de la computación y economía. Recibió el Premio Nobel de Economía, en 1978, por sus investigaciones pioneras en el proceso de toma de decisiones dentro de organizaciones económicas. En lo que respecta al comportamiento humano, es difícil identificar cuál fue el primer trabajo que envolvió el análisis comportamental, pero el hilo de la madeja fue Skinner, con la publicación de su libro, en 1941, Ciencia y Comportamiento Humano (MIROWSKI, 1999).

Otra ciencia que tuvo su origen en las experiencias de guerra fue la Cibernética. Wiener y Julian Bigelow desarrollaron el Previsor Antiaéreo, cuya función consistía en recibir datos de localización (input), sirva como ejemplo, un radar rastreando un avión y retornando a las posibles rutas del avión (output). Esos datos podrían ser utilizados para muchas finalidades, una de ellas, orientar la artillería hacia la posición futura de un avión enemigo. El análisis estadístico era usada para encontrar patrones en esos datos, donde matemática e información eran el núcleo de esa operación. Wiener, al no encontrar ninguna palabra que designara sus ideas, resolvió crearla. Empleó la palabra Cibernética, que derivó de la palabra griega Kubernêtês, que significa piloto o dirigente (WIENER, 1961; GALISON, 1994).

La cibernética parte de la hipótesis de que el modo en que los sistemas, ya sean biológicos, tecnológicos o sociales, responden a los mensajes del mundo exterior son equivalentes y reductibles a modelos matemáticos. La amplitud de ese concepto favoreció el surgimiento de otras ciencias cibernéticas por naturaleza, como la Ciencia Cognitiva, la Inteligencia Artificial, la Robótica y la Informática (WIENER, 1961; GALISON, 1994).

Para la IO, otro factor determinante fue el perfeccionamiento de técnicas, por parte de los científicos que habían trabajado durante la Guerra con IO y estaban motivados a dar continuidad a su trabajo. Uno de esos ejemplos es el método simplex para resolver problemas de programación lineal, desarrollado por George Dantzig (1991). La esfera de cualificaciones de la IO es expandida para incluir nuevas técnicas matemáticas, como la teoría de los juegos y la simulación de Monte Carlo (WIENER, 1961; GALISON, 1994; MIROWSKI, 1999).

Como ya se vio, la IO es un cuerpo interdisciplinar originado en las aplicaciones militares durante la Segunda Guerra Mundial. Sin embargo, su aplicación en otras actividades ha acelerado su desarrollo. Inglaterra ha utilizado la IO en el área de la planificación social y económica del gobierno, la cual puede suministrar previsiones útiles y cuantitativas a la adopción de medidas gubernamentales. En el sector privado, las organizaciones enfrentan un mercado competitivo y su agilidad, para responder a esas demandas y oportunidades depende, cada vez más, de los sistemas de informaciones. Es necesario que los procesos de negocios estén en armonía con las metas y estrategias de la empresa, así es posible disponibilizar 
informaciones y soluciones de calidad en tiempo hábil. La IO es una alternativa, pudiendo suministrar a los ejecutivos elementos cuantitativos para la toma de decisión y en tiempo adecuado, con la ayuda de los computadores (TREFTHEN, 1966).

El desarrollo de las sociedades de IO que tuvo su inicio a partir de los cursos impartidos en las universidades. Era inevitable que la IO hallara el camino de las universidades. El MIT, en colaboración con la Marina, organizó, en 1948, un curso sobre las aplicaciones de la IO y, en 1949, otro que fue realizado en el University College de Londres, compuesto por diez conferencias. Al siguiente año, en la Universidad de Birmingham, tuvo lugar un curso de verano sobre Estudios del Trabajo e Investigación Operacional. El Instituto Tecnológico Case, en 1951, realizó una conferencia sobre las aplicaciones de IO a problemas del comercio y de la industria $\mathrm{y}$, desde entonces, fue la primera institución de enseñanza superior en ofertar un curso de IO y en conferir el título de Licenciado en Ciencias.

En lo que atañe a la literatura y a la propagación de ese conocimiento, presentados a continuación, ingleses y americanos que tuvieron contacto con la IO, durante o después de la guerra, crearon sociedades de IO para el cambio de informaciones y experiencias. En 1948, en Inglaterra, algunos practicantes fundaron el Club de Investigación Operacional, que viene, desde entonces, promoviendo cerca de seis encuentros por año. Fue ese Club el que organizó el curso del University College, ya citado, y lanzó la revista The Operational Research Quarterly, que contenía resúmenes de artículos relevantes para la comunidad de IO (GASS; ASSAD, 2005).

En Estados Unidos, el Consejo Nacional de Investigaciones fundó, en 1949, un Comité de Investigación Operacional y, bajo la coordinación de Horace Levinson, publicó, en 1951, Operations Research with Special Reference to Non-Military Applications, que describía los problemas de esta área y los requisitos para sus profesionales (LEVINSON, 1951). En 1952, en la ciudad de Cambridge, localizada en el estado americano de Massachusetts, fue creada la primera organización profesional de IO, constituyendo la Operational Research Society of America, teniendo a Philip M. Morse como presidente. El objetivo de esa sociedad era establecer y mantener los patrones de cualificación y competencia, mejorar métodos y técnicas y estimular a los estudiantes interesados en esa área.

A partir de 1952, fueron creadas, en varios países, nuevas sociedades con el objeto de desarrollar la IO como una ciencia unificada y promover su avance por todas las naciones. De la cooperación profesional entre los miembros de esas sociedades fue creada a International Federation of Operational Research Societies (IFORS), formada por las Sociedades de Investigación Operacional, actualmente compuesta por 48 países. Es más, la creación de 
primera Federación Internacional de las Sociedades de Investigación Operacional (IFORS), organizada en Oxford, en 1957, fue considerada como un momento decisivo en la historia de la IO y pasó a existir oficialmente en enero de 1959 (MORSE; KIMBALL, 2012).

\section{Consideraciones finales}

En esa investigación retroactiva desde lo siglo de las luces a la modernidad, se puede constatar, por medio de la historia de la IO, que la racionalidad instaurada por la modernidad envuelve actividades racionales, científicas y tecnológicas. Asimismo, por los caminos de su historia fue posible rescatar un acervo rico de temas para discusiones y reflexiones en el mundo actual, tales como: la interdisciplinaridad; las interacciones entre resolución de problemas prácticos e investigación; valorización de la matematización con la globalización de procedimientos y métodos; la matemática como fundadora de la racionalidad moderna; el modelado matemático; como el conocimiento científico puede ser moldeado por los intereses políticos y sociales, entre otros.

Otro factor preponderante en la historia de la IO eran cuestiones relativas a los cambios provocados por el conocimiento científico, en especial el matemático, en las organizaciones sociales y militares, durante y después del régimen de la Segunda Guerra Mundial. Esos cambios ligados a la transferencia de tecnologías de esfuerzo militar para la planificación social después de la guerra cambió el curso de la historia. Dada la importancia del papel asumido por la matemática durante las guerras, surge un nuevo campo de investigación - Matemática y Guerra.

Durante varias décadas, la implicación de la ciencia con la guerra ha provocado debates radicales sobre su naturaleza y el papel asumido por la comunidad científica, en lo que concierne a su implicación con la guerra. Hay una conciencia generalizada de que la matemática es una de las ciencias provocadoras de ese debate, debido a su contribución a la industria bélica y a las estrategias de guerra. Esa relación suscita una doble interpretación.

En cierto sentido, se puede cuestionar la importancia de la matemática para el armamento de guerra y, en el sentido opuesto, la importancia de los armamentos de guerra para el desarrollo matemático. En el primer caso, el fruto de esa relación es bastante evidente. La propia historia de la IO muestra la importancia de la matemática para el desarrollo de las tecnologías bélicas. En el segundo caso, puede ser constatado, con el régimen que se configuró durante la Segunda Guerra Mundial. Fue un periodo de intenso desarrollo científico y 
tecnológico, con la presencia masiva del gobierno federal americano en la planificación y financiación de la ciencia.

Las universidades, como instituciones, fueron fortalecidas, pues eran los canales a través de los cuales los contratos eran firmados con los científicos, en consonancia con la planificación y política científica. Las industrias también fueron utilizadas con esa misma finalidad. Varios proyectos fueron creados con ese propósito y el mayor de ellos fue el proyecto de la construcción de la bomba atómica, el proyecto Manhattan, el cual empleó 130.000 personas, en 1945.

Cualquier ciencia aplicada a resolver problemas bélicos exige una discusión ética bastante proficua, sobre todo tras el surgimiento de la bomba atómica, sin embargo, para que esa reflexión sea posible, la historia de cómo las estrategias militares se valieron de la ciencia para tales fines, es necesaria y debe ser revisada siempre. Sólo así, el hombre puede tener la perspectiva de cómo, cuándo, dónde y, principalmente, para qué fines utilizar la ciencia.

\section{Referencias}

ACKOFF, R. L.; SASIENI, M. W. Pesquisa Operacional. 1. ed. Rio de Janeiro: Livros Técnicos e Científicos, 1971.

ARENALES, M.; ARMENTANO, V.; MORABITO, R; YANASSE, H. Pesquisa operacional: para cursos de engenharia. 2. ed São Paulo: Elsevier Brasil, 2015.

BARROS, G. Racionalidade e Organizações: Um Estudo sobre o Comportamento Econômico na Obra de Herbert A. Simon. 2004. 145 f. Dissertação (Mestrado em Economia) - Departamento de Economia da Faculdade de Economia, Administração e Ciências Contábeis, Universidade de São Paulo, São Paulo, 2004. Disponível em <http://www.teses.usp.br/teses/disponiveis/12/12138/tde05032005-183337/en.php>. Acesso em: 01 nov. 2016.

CAMPBELL, V. How Rand Invented The Postwar World, 2004. Disponível em: <http://www.rand. org/content/dam/rand/www/external/about/history/Rand.IT.Summer04.pdf >. Acesso em: 12 jan. 2017.

CHAVES, V. H. C. Perspectivas históricas da pesquisa operacional. 2011. 117 f. Dissertação (Mestrado em Educação Matemática) - Instituto de Geociências e Ciências Exatas, Universidade Estadual Paulista "Júlio de Mesquita Filho", Rio Claro, 2011.

DANTZIG, G. B. Linear Programming. In: LENSTRA, J. K; KAN, A. H. G. R.; SCHRIJVER, A. (Org.). History of Mathematical Programming. New York: Elsevier Science Publishing Company, 1991. p. 19-31.

DUREN, P. et al. A Century of Mathematics in America. 3. ed. Rhode Island: American Mathematical Society Press, 1989.

FORTUN, M.; SCHWEBER, S. S. Scientists and the legacy of World War II: The case of operations research (OR). Social Studies of Science, London, v. 23, n. 4, p. 595-642, 1993.

GALISON, P. The ontology of the enemy: Norbert Wiener and the cybernetic vision. Critical inquiry, Chicago, v. 21, n. 1, p. 228-266, 1994. 
GASS, S. I.; ASSAD, A. A. An annotated timeline of operations research: An informal history. 1. ed. New York: Springer Science \& Business Media, 2005.

GLICK, S. P.; CHARTERS, L. I. War, Games and Military History. Journal of Contemporary History, London, v. 18, n. 4, p. 567-582. oct. 1983.

HENDERSON, L. J. Organização para Pesquisa Operacional. In: TREFETHEN, F. N.; MCCLOSKEY, J. F. (Org.). Pesquisa Operacional: como instrumento de gerência. Tradução de Léo Magarinos de Souza Leão. Rio de Janeiro: USAID, 1966. p. 86-98.

HILLIER, F. S.; LIEBERMAN, G. J. Introdução à pesquisa operacional. 9. ed. Porto Alegre: McGraw Hill Brasil, 2013.

JOHNSON, E. A. A Direção Executiva, a Organização e a Pesquisa Operacional. In: TREFETHEN, F. N.; MCCLOSKEY, J. F. (Org.). Pesquisa Operacional: como instrumento de gerência. Tradução de Léo Magarinos de Souza Leão. Rio de Janeiro: USAID, 1966. p. 13-24.

KIRBY, M. W. Operational research in war and peace: the British experience from the 1930s to 1970. 1. ed. London: Imperial College Press, 2003.

LEVINSON, H. C. Operations Research with Special Reference to Non-military Applications: A Brochure. 1. ed. Washington D.C.: National Academies, 1951.

MCARTHUR, C. W. Operations Analysis in the US Army Eighth Air Force in World War II. 1. ed. Rhode Island: American Mathematical Soc., 1990.

MCCLOSKEY, J. F. US operations research in World War II. Operations Research, Catonsville, v. 35, n. 6, p. 910-925, 1987.

MIGUEL, A. Pesquisa em Educação Matemática e mentalidade bélica. Bolema, Rio Claro, n. 25, p. 116, 2006.

MIROWSKI, P. Cyborg Agonistes: Economics Meets Operations Research in Mid- Century. Social Studies of Science, London, v. 29, p. 685-718, oct. 1999.

MIROWSKI, P.; SENT, E. Science Bought and Sold: Essays in Economics of Science. 1. ed. Chicago: The University of Chicago Press, 2002.

MORSE, P. M. The beginnings of operations research in the United States. Operations Research, Catonsville, v. 34, n. 1, p. 10-17, 1986.

MORSE, P. M.; KIMBALL, G. E. Methods of operations research. 3. ed. Local: Courier Corporation, 2012.

RANGER, R. Theater missile defenses: Lessons from British experiences with air and missile defenses. Comparative Strategy, UK, v. 12, n. 4, p. 399-413, 1993.

ROWE, A. P. One story of radar. 2. ed. Cambridge: Cambridge University Press, 2015.

SERRES, M. Hominescências. 1. ed. São Paulo: Bertrand Brasil, 2003.

SIMON, H. A. The Shape of Automation: for Men and Management. 1. ed. New York: Harper \& Row, 1965. 
TREFETHEN, F. N. História da Pesquisa Operacional. In: TREFETHEN, F. N.; MCCLOSKEY, J. F. (Org.). Pesquisa Operacional: como instrumento de gerência. Tradução de Léo Magarinos de Souza Leão. Rio de Janeiro: USAID, 1966. p. 27-57.

TREST, W. A. Air Force Roles and Missions: A History. 1. ed. Washington D.C.: Government Printing Office, 1998.

VON NEUMANN, J.; MORGENSTERN, O. The Theory of Games and Economic Behavior. 1. ed. Princeton: Princeton University Press, 1953.

WIENER, N. Cybernetics: or control and communication in the animal and the machine. 1. ed. Cambridge, Massachusetts: M. I. T. Press, 1961.

Submetido em 14 de Setembro de 2017. Aprovado em 18 de Dezembro de 2017. 